Baogang Wang and Xiaxia Man*

\title{
Crystal structure of poly[triaqua-(di(2,2'-bipyridine- $\left.{ }^{2} N, N^{\prime}\right)-\mu_{4^{-}}$ silanetetrayltetrakis(benzene-4,1-diyl)tetrakis (hydrogen

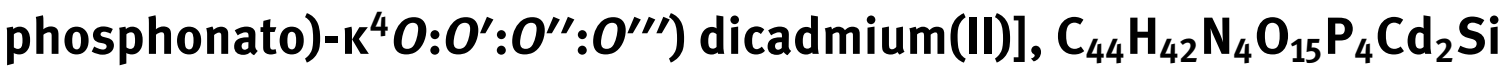

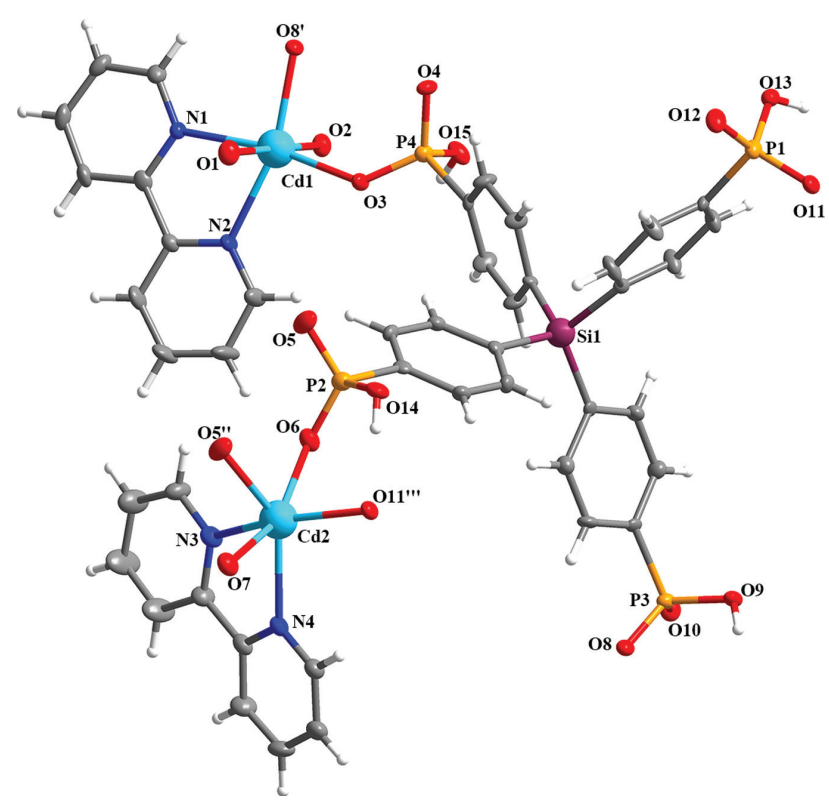

https://doi.org/10.1515/ncrs-2020-0316

Received June 28, 2020; accepted August 20, 2020; available online September 12, 2020

\section{Abstract} $b=14.2036(9) \AA, \quad c=15.2206(10) \AA, \quad \alpha=69.637(1)^{\circ}$, $\beta=72.355(1)^{\circ}, \quad \gamma=64.861(1)^{\circ}, \quad V=2431.1(3) \AA^{3}, \quad Z=2$, $R_{\mathrm{gt}}(F)=0.0428, w R_{\text {ref }}\left(F^{2}\right)=0.0987, T=120(2) \mathrm{K}$.

\section{CCDC no.: 2024330}

A part of the title crystal structure is shown in the figure. Tables 1 and 2 contain details of the measurement method and a list of the atoms including atomic coordinates and displacement parameters.

*Corresponding author: Xiaxia Man, The Department of Oncological Gynecology, The First Hospital of Jilin University, Changchun, Jilin, 130021, China, e-mail:drmanxx@protonmail.com. https://orcid. org/0000-0001-7221-7119

Baogang Wang: The Department of Cardiac Surgery, The First Hospital of Jilin University, Changchun, Jilin, 130021, China
$\mathrm{C}_{44} \mathrm{H}_{42} \mathrm{~N}_{4} \mathrm{O}_{15} \mathrm{P}_{4} \mathrm{Cd}_{2} \mathrm{Si}$, triclinic, $P \overline{1}$ (no. 2), $a=13.4778$ (9) $\AA$,

Table 1: Data collection and handling.

\begin{tabular}{ll}
\hline Crystal: & Colorless block \\
Size: & $0.50 \times 0.40 \times 0.30 \mathrm{~mm}$ \\
Wavelength: & Mo $K \alpha$ radiation $(0.71073 \AA)$ \\
$\mu:$ & $1.10 \mathrm{~mm}^{-1}$ \\
Diffractometer, scan mode: & Bruker D8 QUEST, $\varphi$ and $\omega$ \\
$\theta_{\max }$, completeness: & $26.1^{\circ}, 99 \%$ \\
$N(h k l)_{\text {measured }}, N(h k l)_{\text {unique }}, R_{\text {int }}:$ & $15498,9617,0.034$ \\
Criterion for $I_{\text {obs }}, N(h k l)_{\mathrm{gt}}:$ & $I_{\text {obs }}>2 \sigma\left(I_{\text {obs }}\right), 7610$ \\
$N(\text { param })_{\text {refined }}:$ & 643 \\
Programs: & Bruker [1], SHELX [2, 3] \\
\hline
\end{tabular}

Table 2: Fractional atomic coordinates and isotropic or equivalent isotropic displacement parameters $\left(\AA^{2}\right)$.

\begin{tabular}{lrrrr}
\hline Atom & $\boldsymbol{x}$ & $\boldsymbol{y}$ & $\boldsymbol{z}$ & $\boldsymbol{U}_{\text {iso }}{ }^{*} \boldsymbol{U}_{\text {eq }}$ \\
\hline C1 & $0.4668(3)$ & $-0.2011(3)$ & $1.1643(3)$ & $0.0144(9)$ \\
C2 & $0.5800(4)$ & $-0.2208(4)$ & $1.1481(3)$ & $0.0261(11)$ \\
H2 & 0.6234 & -0.2254 & 1.0865 & $0.031^{*}$ \\
C3 & $0.6306(4)$ & $-0.2340(4)$ & $1.2205(3)$ & $0.0251(11)$ \\
H3 & 0.7084 & -0.2481 & 1.2080 & $0.030^{*}$ \\
C4 & $0.5696(3)$ & $-0.2270(3)$ & $1.3108(3)$ & $0.0148(9)$ \\
C5 & $0.4577(4)$ & $-0.2108(4)$ & $1.3286(3)$ & $0.0238(10)$ \\
H5 & 0.4148 & -0.2078 & 1.3905 & $0.029^{*}$ \\
C6 & $0.4074(4)$ & $-0.1990(4)$ & $1.2557(3)$ & $0.0219(10)$ \\
H6 & 0.3305 & -0.1894 & 1.2693 & $0.026^{*}$ \\
C7 & $0.8206(5)$ & $0.0278(4)$ & $1.2278(3)$ & $0.0345(13)$ \\
H7 & 0.7902 & -0.0160 & 1.2174 & $0.041^{*}$ \\
C8 & $0.8642(5)$ & $0.0930(5)$ & $1.1503(4)$ & $0.0435(16)$ \\
H8 & 0.8655 & 0.0933 & 1.0875 & $0.052^{*}$ \\
C9 & $0.9061(5)$ & $0.1581(5)$ & $1.1651(4)$ & $0.0391(14)$ \\
H9A & 0.9363 & 0.2045 & 1.1127 & $0.047^{*}$ \\
C10 & $0.9036(4)$ & $0.1549(4)$ & $1.2570(4)$ & $0.0295(11)$ \\
H10 & 0.9320 & 0.1994 & 1.2687 & $0.035^{*}$ \\
C11 & $0.8595(4)$ & $0.0865(3)$ & $1.3326(3)$ & $0.0186(9)$ \\
C12 & $0.8542(3)$ & $0.0794(3)$ & $1.4341(3)$ & $0.0183(9)$ \\
C13 & $0.9024(4)$ & $0.1348(3)$ & $1.4586(3)$ & $0.0232(10)$ \\
H13A & 0.9365 & 0.1814 & 1.4105 & $0.028^{*}$ \\
C14 & $0.8994(4)$ & $0.1205(4)$ & $1.5539(4)$ & $0.0269(11)$ \\
H14A & 0.9309 & 0.1578 & 1.5722 & $0.032^{*}$ \\
C15 & $0.8499(4)$ & $0.0515(4)$ & $1.6223(3)$ & $0.0260(11)$ \\
H15A & 0.8487 & 0.0389 & 1.6880 & $0.031^{*}$ \\
C16 & $0.8024(4)$ & $0.0015(4)$ & $1.5930(3)$ & $0.0232(10)$ \\
H16 & 0.7666 & -0.0444 & 1.6400 & $0.028^{*}$ \\
C17 & $0.3003(3)$ & $-0.0155(3)$ & $1.0484(3)$ & $0.0164(9)$ \\
C18 & $0.3027(4)$ & $0.0489(3)$ & $1.0981(3)$ & $0.0187(9)$ \\
H18 & 0.3519 & 0.0186 & 1.1423 & $0.022^{*}$ \\
C19 & $0.2350(4)$ & $0.1563(4)$ & $1.0845(3)$ & $0.0191(10)$ \\
& & & &
\end{tabular}

๖ Open Access. ( 2020 Baogang Wang et al., published by De Gruyter. (c) BY This work is licensed under the Creative Commons Attribution 4.0 International License. 
Table 2 (continued)

\begin{tabular}{|c|c|c|c|c|}
\hline Atom & $x$ & $y$ & $z$ & $U_{\text {iso }}{ }^{\star} / U_{\text {eq }}$ \\
\hline H19 & 0.2382 & 0.1985 & 1.1194 & $0.023^{*}$ \\
\hline $\mathrm{C} 20$ & $0.1627(3)$ & $0.2030(3)$ & $1.0206(3)$ & $0.0174(9)$ \\
\hline $\mathrm{C} 21$ & $0.1609(4)$ & $0.1401(4)$ & $0.9687(3)$ & $0.0230(10)$ \\
\hline $\mathrm{H} 21$ & 0.1130 & 0.1709 & 0.9235 & 0.028 * \\
\hline $\mathrm{C} 22$ & $0.2288(4)$ & $0.0332(4)$ & $0.9831(3)$ & $0.0220(10)$ \\
\hline H22 & 0.2267 & -0.0086 & 0.9471 & $0.026^{\star}$ \\
\hline $\mathrm{C} 23$ & $0.2830(3)$ & $-0.2358(3)$ & $1.1210(3)$ & $0.0156(9)$ \\
\hline C24 & $0.3185(4)$ & $-0.3464(4)$ & $1.1536(3)$ & $0.0250(11)$ \\
\hline H24 & 0.3959 & -0.3873 & 1.1425 & $0.030^{*}$ \\
\hline $\mathrm{C} 25$ & $0.2441(4)$ & $-0.3993(4)$ & $1.2022(3)$ & $0.0253(11)$ \\
\hline $\mathrm{H} 25$ & 0.2712 & -0.4755 & 1.2241 & $0.030^{\star}$ \\
\hline C26 & $0.1304(3)$ & $-0.3417(3)$ & $1.2191(3)$ & $0.0155(9)$ \\
\hline $\mathrm{C} 27$ & $0.0935(4)$ & $-0.2311(4)$ & $1.1869(4)$ & $0.0276(11)$ \\
\hline $\mathrm{H} 27$ & 0.0160 & -0.1903 & 1.1982 & $0.033^{\star}$ \\
\hline $\mathrm{C} 28$ & $0.1692(4)$ & $-0.1790(4)$ & $1.1379(3)$ & $0.0258(11)$ \\
\hline $\mathrm{H} 28$ & 0.1422 & -0.1028 & 1.1156 & $0.031^{\star}$ \\
\hline C29 & $0.4789(4)$ & $-0.1921(3)$ & $0.9552(3)$ & $0.0162(9)$ \\
\hline C30 & $0.5577(4)$ & $-0.2933(3)$ & $0.9488(3)$ & $0.0211(10)$ \\
\hline H3O & 0.5663 & -0.3506 & 1.0050 & $0.025^{\star}$ \\
\hline C31 & $0.6239(4)$ & $-0.3128(4)$ & $0.8630(3)$ & $0.0218(10)$ \\
\hline H31 & 0.6776 & -0.3824 & 0.8610 & $0.026^{*}$ \\
\hline C32 & $0.6117(3)$ & $-0.2301(3)$ & $0.7794(3)$ & $0.0156(9)$ \\
\hline C33 & $0.5370(4)$ & $-0.1287(4)$ & $0.7847(3)$ & $0.0220(10)$ \\
\hline H33 & 0.5292 & -0.0714 & 0.7285 & $0.026^{\star}$ \\
\hline C34 & $0.4734(4)$ & $-0.1098(4)$ & $0.8711(3)$ & $0.0223(10)$ \\
\hline H34 & 0.4244 & -0.0387 & 0.8735 & $0.027^{\star}$ \\
\hline C35 & $0.2608(4)$ & $0.5739(4)$ & $0.8784(3)$ & $0.0309(12)$ \\
\hline H35 & 0.2143 & 0.5546 & 0.9371 & $0.037^{\star}$ \\
\hline C36 & $0.3456(5)$ & $0.6031(5)$ & $0.8789(4)$ & $0.0448(15)$ \\
\hline H36 & 0.3581 & 0.6033 & 0.9370 & $0.054^{\star}$ \\
\hline C37 & $0.4122(5)$ & $0.6321(6)$ & $0.7933(4)$ & $0.0546(18)$ \\
\hline H37 & 0.4697 & 0.6549 & 0.7917 & $0.065^{\star}$ \\
\hline C38 & $0.3949(5)$ & $0.6277(5)$ & $0.7100(4)$ & $0.0467(16)$ \\
\hline H38 & 0.4417 & 0.6453 & 0.6509 & $0.056^{\star}$ \\
\hline C39 & $0.3081(4)$ & $0.5971(4)$ & $0.7139(3)$ & $0.0275(11)$ \\
\hline $\mathrm{C} 40$ & $0.2825(4)$ & $0.5908(4)$ & $0.6288(3)$ & $0.0241(10)$ \\
\hline C41 & $0.1611(4)$ & $0.5688(4)$ & $0.5646(3)$ & $0.0258(11)$ \\
\hline H41 & 0.0940 & 0.5573 & 0.5730 & $0.031^{\star}$ \\
\hline C42 & $0.2252(4)$ & $0.5815(4)$ & $0.4744(3)$ & $0.0317(12)$ \\
\hline H42 & 0.2027 & 0.5790 & 0.4221 & $0.038^{\star}$ \\
\hline C43 & $0.3229(4)$ & $0.5978(4)$ & $0.4623(3)$ & $0.0344(12)$ \\
\hline H43 & 0.3702 & 0.6046 & 0.4016 & $0.041^{*}$ \\
\hline C44 & $0.3508(4)$ & $0.6040(4)$ & $0.5394(3)$ & $0.0316(12)$ \\
\hline H44 & 0.4168 & 0.6173 & 0.5317 & $0.038^{\star}$ \\
\hline $\mathrm{Cd} 1$ & $0.73028(2)$ & $-0.07422(2)$ & $1.44706(2)$ & $0.01366(8)$ \\
\hline $\mathrm{Cd} 2$ & $0.08702(3)$ & $0.53987(3)$ & $0.79651(2)$ & $0.01772(9)$ \\
\hline $\mathrm{H} 7 \mathrm{~A}$ & $-0.090(3)$ & $0.705(4)$ & $0.699(3)$ & $0.032(15)^{\star}$ \\
\hline H7B & $-0.073(5)$ & $0.722(5)$ & $0.776(5)$ & $0.07(2)^{\star}$ \\
\hline N1 & $0.8045(3)$ & $0.0146(3)$ & $1.5012(2)$ & $0.0165(8)$ \\
\hline $\mathrm{N} 2$ & $0.8190(3)$ & $0.0233(3)$ & $1.3174(3)$ & $0.0193(8)$ \\
\hline N3 & $0.2413(3)$ & $0.5717(3)$ & $0.7983(3)$ & $0.0261(9)$ \\
\hline N4 & $0.1885(3)$ & $0.5718(3)$ & $0.6406(3)$ & $0.0220(8)$ \\
\hline 01 & $0.5709(2)$ & $0.0764(3)$ & $1.4316(2)$ & $0.0250(7)$ \\
\hline $\mathrm{H} 1 \mathrm{~A}$ & 0.5482 & 0.1258 & 1.4620 & $0.038^{\star}$ \\
\hline H1B & 0.5101 & 0.0770 & 1.4222 & $0.038^{\star}$ \\
\hline 02 & $0.8981(2)$ & $-0.2259(2)$ & $1.4616(2)$ & $0.0213(7)$ \\
\hline
\end{tabular}

Table 2 (continued)

\begin{tabular}{|c|c|c|c|c|}
\hline Atom & $x$ & $y$ & $z$ & $U_{\text {iso }} * / U_{\text {eq }}$ \\
\hline $\mathrm{H} 2 \mathrm{~A}$ & 0.9110 & -0.2599 & 1.4193 & $0.032^{*}$ \\
\hline $\mathrm{H} 2 \mathrm{~B}$ & 0.8889 & -0.2717 & 1.5161 & $0.032^{*}$ \\
\hline 03 & $0.6632(2)$ & $-0.1350(2)$ & $1.3723(2)$ & $0.0186(7)$ \\
\hline 04 & $0.5531(2)$ & $-0.2500(2)$ & $1.49598(19)$ & $0.0182(6)$ \\
\hline 05 & $0.0411(3)$ & $0.3686(2)$ & $1.0959(2)$ & $0.0296(8)$ \\
\hline 06 & $0.1418(2)$ & $0.4047(2)$ & $0.9214(2)$ & $0.0227(7)$ \\
\hline 07 & $-0.0447(3)$ & $0.7146(3)$ & $0.7212(3)$ & $0.0255(8)$ \\
\hline 08 & $0.6554(2)$ & $-0.1430(2)$ & $0.59366(19)$ & $0.0175(6)$ \\
\hline 09 & $0.6317(2)$ & $-0.3243(2)$ & $0.6481(2)$ & $0.0182(6)$ \\
\hline H9 & 0.6071 & -0.2952 & 0.5969 & $0.027^{\star}$ \\
\hline 010 & $0.8064(2)$ & $-0.3139(2)$ & $0.6611(2)$ & $0.0214(7)$ \\
\hline 011 & $0.0118(2)$ & $-0.4517(2)$ & $1.2162(2)$ & $0.0205(7)$ \\
\hline 012 & $-0.0712(3)$ & $-0.3240(2)$ & $1.3244(2)$ & $0.0257(7)$ \\
\hline 013 & $0.0822(2)$ & $-0.4976(2)$ & $1.3671(2)$ & $0.0208(7)$ \\
\hline H13 & 0.1163 & -0.5551 & 1.3498 & 0.031 * \\
\hline 014 & $-0.0284(2)$ & $0.3540(2)$ & $0.9683(2)$ & $0.0228(7)$ \\
\hline H14 & -0.0255 & 0.3847 & 0.9099 & $0.034^{*}$ \\
\hline 015 & $0.7389(2)$ & $-0.3380(2)$ & $1.4079(2)$ & $0.0233(7)$ \\
\hline H15 & 0.7955 & -0.3222 & 1.3777 & $0.035^{\star}$ \\
\hline P1 & $0.02951(9)$ & $-0.40560(9)$ & $1.28371(8)$ & $0.0165(2)$ \\
\hline P2 & $0.07746(10)$ & $0.34409(9)$ & $1.00062(8)$ & $0.0192(3)$ \\
\hline P3 & $0.68338(9)$ & $-0.25233(9)$ & $0.66279(7)$ & $0.0144(2)$ \\
\hline P4 & $0.63298(9)$ & $-0.23372(9)$ & $1.40194(8)$ & $0.0153(2)$ \\
\hline Si1 & $0.38576(10)$ & $-0.16328(9)$ & $1.06988(8)$ & $0.0142(2)$ \\
\hline
\end{tabular}

A mixture of silanetetrayltetrakis(benzene-4,1-diyl)tetrakis phosphonic acid $(0.2 \mathrm{mmol}), 2,2^{\prime}$-bipyridine $(0.2 \mathrm{mmol})$, $\mathrm{Cd}\left(\mathrm{NO}_{3}\right)_{2} \cdot 4\left(\mathrm{H}_{2} \mathrm{O}\right)(0.2 \mathrm{mmol}), \mathrm{H}_{2} \mathrm{O}(8 \mathrm{~mL})$ was stirred for ten minutes. The mixture was transferred in a $25 \mathrm{~mL}$ stainless steel reactor with a Teflon liner and heated from 298 to $453 \mathrm{~K}$ in $5 \mathrm{~h}$ and a constant temperature was maintained at $453 \mathrm{~K}$ for $72 \mathrm{~h}$. After cooling to room temperature, colorless block crystals were collected in 55.3\% yield based on the amounts of Cd.

\section{Experimental details}

Coordinates of hydrogen atoms were calculated. Their $U_{\text {iso }}$ values were set to $1.2 U_{\text {eq }}$ of the parent atoms.

\section{Comment}

Coordination polymers ( $\mathrm{CPs}$ ) composed of metal nodes and bridging linkers have attracted much attention over the past decades due to their enormous variety of intriguing structural topologies and great potential applications [4, 5]. Compared with carboxylates and pyrazolate counterparts, phosphonate groups have a stronger interaction with metal ions, and consequently higher chemical and thermal stability [6-8]. The excellent robustness of metal phosphonates represents 
remarkable added value and should promote their investigation, regardless of the challenges relating to their synthesis and characterization, in order to develop a new generation of robust CPs able to overcome the typical weaknesses of classical CPs.

The asymmetric unit of the title structure contains two crystallographically independent cadmium(II) ions, one silanetetrayltetrakis (benzene-4,1-diyl)tetrakis(hydrogen phosphonato) ligand, two 2,2'-bipyridine and three coordinated water molecules. Cd1 is six-coordinated by four $\mathrm{O}$ atoms from two silanetetrayltetrakis(benzene-4,1-diyl) tetrakis(hydrogen phosphonato) ligands and two water molecules, two $\mathrm{N}$ atoms from one 2,2'-bipyridine, forming a distorted octahedral geometry. $\mathrm{Cd} 2$ is also six-coordinated by four $\mathrm{O}$ atoms from three silanetetrayltetrakis(benzene-4,1diyl)tetrakis(hydrogen phosphonato) ligands and one water molecule, two $\mathrm{N}$ atoms from one 2,2'-bipyridine, also forming a distorted octahedral geometry. The distances between $\mathrm{Cd}$ and the coordinated atoms are: $d(\mathrm{Cd} 1 \cdots 01)=2.302(3) \AA$, $\mathrm{d}(\mathrm{Cd} 1 \cdots \mathrm{O} 2)=2.375(3) \AA, \quad \mathrm{d}(\mathrm{Cd} 1 \cdots \mathrm{O} 3)=2.199(3) \AA$, $\mathrm{d}(\mathrm{Cd} 1 \cdots \mathrm{O} 8)=2.215(3) \AA, \quad \mathrm{d}(\mathrm{Cd} 1 \cdots \mathrm{N} 1)=2.360(3) \AA$, $\mathrm{d}(\mathrm{Cd} 1 \cdots \mathrm{N} 2)=2.360(3) \AA, \quad \mathrm{d}(\mathrm{Cd} 2 \cdots \mathrm{O} 5)=2.284(3) \AA$, $\mathrm{d}(\mathrm{Cd} 2 \cdots \mathrm{O}$. $)=2.224(3) \AA, \quad \mathrm{d}(\mathrm{Cd} 2 \cdots 07)=2.477(3) \AA$, $\mathrm{d}(\mathrm{Cd} 2 \cdots \mathrm{O} 11)=2.264(3) \AA \mathrm{d}(\mathrm{Cd} 2 \cdots \mathrm{N} 3)=2.317(4) \AA$, and $\mathrm{d}(\mathrm{Cd} 2 \cdots \mathrm{N} 4)=2.349(4) \AA$, respectively. The Cd1 coordination angles are from $70.93(12)^{\circ}$ to $178.12(10)^{\circ}$, and $\mathrm{Cd} 2$ coordination angles are from $66.81(12)^{\circ}$ to $158.45(12)^{\circ}$. These above mentioned bond distances and angles are all in their normal ranges. They can be compared with those previously reported compounds [9]. The Cd1 ion links its symmetryrelated atoms to form a dinuclear secondary building unit (SBU) bridged by two silanetetrayltetrakis(benzene-4,1-diyl) tetrakis (hydrogen phosphonato) ligands. Cd2 ion is mononuclear linked by two silanetetrayltetrakis (benzene-4,1-diyl) tetrakis(hydrogen phosphonato) ligands. The Cd1 dinuclear SBUs and Cd2 mononuclear are linked by silanetetrayltetrakis(benzene-4,1-diyl)tetrakis (hydrogen phosphonato) ligands to form two-dimensional (2D) layer framework parallel to the $b c$ plane. These 2D layers are packed together to form 3D framework structures through the $\pi-\pi$ interactions between neighboring benzene rings from parallel 2,2'-bipyridine. There are related structures that have been reported with the same ligand [10]. For example, threedimensional compound $\mathrm{Cd}_{4}\left(\mathrm{H}_{4} \mathrm{~L}\right)_{2}(\text { phen })_{2}\left(\mathrm{H}_{2} \mathrm{O}\right)_{4}$ which is characterized as a 3,4,6-connected network with the vertex symbol of $\left\{4 \cdot 6^{4} \cdot 8\right\}^{2}\left\{4^{2} \cdot 6^{4} \cdot 8^{9}\right\}\left\{6^{2} \cdot 8\right\}^{2}$ and the compound $\mathrm{Cu}_{2} \mathrm{H}_{4} \mathrm{STPPA} \cdot 2 \mathrm{H}_{2} \mathrm{O}$ with three dimensional porous framework were synthesized using hydrothermal reaction method $[11,12]$.

Acknowledgements: This work is supported by the project development plan of science and technology of Jilin Province.

\section{References}

1. Bruker. APEX2, SAINT and SADABS. Bruker AXS Inc., Madison, WI, USA (2009).

2. Sheldrick, G. M.: SHELXT - Integrated space-group and crystalstructure determination. Acta Crystallogr. A71 (2015) 3-8.

3. Sheldrick, G. M.: Crystal structure refinement with SHELXL. Acta Crystallogr. C71 (2015) 3-8.

4. Pettinari, C.; Tabacaru, A.; Galli, S.: Coordination polymers and metal-organic frameworks based on poly(pyrazole)-containing ligands. Coord. Chem. Rev. 307 (2016) 1-31.

5. Yang, X.; Zhang, Y.; Li, F.; Guo, T.; Wu, Y.; Jin, F.; Fang, M.; Lan, Y.; Li, Y.; Zhou, Y.; Zou, Z.: Theoretical and experimental studies on three water-stable, isostructural, paddlewheel based semiconducting metal-organic frameworks. Dalton Trans. 46 (2017) 8204-8218.

6. Taddei, M.; Sassi, P.; Costantino, F.; Vivani, R.: Aminofunctionalized layered crystalline zirconium phosphonates: synthesis, crystal structure, and spectroscopic characterization. Inorg. Chem. 55 (2016) 6278-6285.

7. Yücesan, G.; Zorlu, Y.; Stricker, M.; Beckmann, J.: Metal-organic solids derived from arylphosphonic acids. Coord. Chem. Rev. 369 (2018) 105-122.

8. Taddei, M.; Costantino, F.; Vivani, R.: Robust metal-organic frameworks based on tritopic phosphonoaromatic ligands. Eur. J. Inorg. Chem. 2016 (2016) 4300-4309.

9. Hasegawa, Y.; Kitagawa, Y.: Thermo-sensitive luminescence of lanthanide complexes, clusters, coordination polymers and metal-organic frameworks with organic photosensitizers. J. Mater. Chem. C 7 (2019) 7494-7511.

10. Liu, Z.; You, P.; Tang, M.; Zhang, L.; Liu, S.: The crystal structure of dimethyl ((3,5-di-tert-butyl-4-hydroxyphenyl)(phenyl) methyl) phosphonate, $\mathrm{C}_{23} \mathrm{H}_{33} \mathrm{O}_{4}$ P. Z. Kristallogr. NCS 234 (2019) 865-867.

11. Schütrumpf, A.; Bulut, A.; Hermer, N.; Zorlu, Y.; Kirpi, E.; Stock, N.; Yazaydın, A.; Yücesan, G.; Beckmann, J.: From tetrahedral tetraphosphonic acids $\mathrm{E}\left[\mathrm{p}-\mathrm{C}_{6} \mathrm{H}_{4} \mathrm{P}(\mathrm{O})(\mathrm{OH})_{2}\right]_{4}(\mathrm{E}=\mathrm{C}$, $\mathrm{Si})$ to porous $\mathrm{Cu}$ - and $\mathrm{Zn}$-MOFs with large surface areas. ChemistrySelect 2 (2017) 3035-3038.

12. Ai, J.; Tian, H.-R.; Min, X.; Wang, Z.-C. Sun, Z.-M.: A fast and highly selective Congo red adsorption material based on a cadmium-phosphonate network. Dalton Trans. 49 (2020) 3700-3705. 